
\title{
25 Research Square \\ TAZ Regulates Bladder Cancer Cell Proliferation and Apoptosis via p38 Activity
}

\section{Yangyang Sun}

The First Affiliated Hospital of Shenzhen University https://orcid.org/0000-0002-4357-611X

\section{Lianxin $\mathrm{Hu}$}

The First Affiliated Hospital of Shenzhen University

\section{Chengxi Liu}

Shanghai Jiao Tong University

\section{Ganglin Su}

Shantou University Medical College

\section{Lina Yang}

Qingdao University

\section{Weiren Huang}

The First Affiliated Hospital of Shenzhen University

\section{Wei Chen ( $\nabla$ weichen@sibcb.ac.cn )}

The First Affiliated Hospital of Shenzhen University

\section{Research article}

Keywords: TAZ, Hippo pathway, p38 MAPK pathway, Bladder cancer

Posted Date: October 26th, 2020

DOI: https://doi.org/10.21203/rs.3.rs-95663/v1

License: (c) (1) This work is licensed under a Creative Commons Attribution 4.0 International License.

Read Full License 


\section{Abstract}

Background The progression of cancer is driven by the deregulation of various signaling pathways, especially Hippo and p38 MAPK pathway. TAZ, a downstream target of Hippo pathway, has been demonstrated to promote tumorigenesis in various cancers, but the functions of both Hippo and p38 signaling in bladder cancer cells are still unclear.

Methods T24 and 5637 cells with knockdown of TAZ were constructed. EdU cell proliferation assay and western blot were used to illustrate the effects of TAZ on the proliferation and apoptosis of bladder cancer cells and the expression of p38 protein and phosphorylation. We overexpressed Flag-tagged TAZ in 293T cells. Western blot and RT-qPCR were used to further illustrate the effect of TAZ on the expression level of p38. The p38 inhibitor (PH-797804) combined with western blot and EDU cell proliferation assay were used to show whether TAZ affects the proliferation and apoptosis of bladder cancer cells by regulating the activity of $\mathrm{p} 38$.

Results The shTAZ contained in T24 and 5637 cells significantly inhibited bladder cancer cells proliferation, in addition, the knockdown of TAZ-induced apoptosis of T24 and 5637 cells was found out. The loss of TAZ led to the upregulation of $\mathrm{p} 38$ protein as well as phosphorylation. The over-expression of Flag-TAZ had no obvious effect on p38 mRNA level, but p38 protein was reduced clearly in 293T cells. ShTAZ induced the upregulation of cleaved-caspase 3, which disappeared when treated with $\mathrm{PH}-797804$, a p38 inhibitor, and the reduction to EdU positive cells induced by shTAZ was reversed by $\mathrm{PH}-797804$ treatment, which suggested that p38 activity could mediate both cell proliferation and apoptosis regulated by TAZ knockdown.

Conclusions In this study, it was demonstrated that TAZ could regulate the proliferation and apoptosis of bladder cancer cells by regulating the stability of p38 protein. Our finding uncovered the novel functional interaction between Hippo and p38 MAPK pathway. An in-depth understanding of this question may indicate a new direction of diagnosis or treatment for bladder cancer.

\section{Background}

Bladder cancer is the most common urological tumor that leads to cancer-related death around the world $[1,2]$. Surgery, radiation therapy and chemotherapy are currently the major therapies for the treatment of patients diagnosed with bladder cancer, but their survival rates are still low ${ }^{[3,4]}$. One of the major reasons is that the molecular mechanism of bladder cancer progression is still unclear.

Generally speaking, the progression of cancer is driven by the deregulation of various signaling pathways related to cell proliferation and apoptosis. Among them, Hippo and p38 MAPK pathway are two crucial ones. Tissue growth and organ size are controlled by Hippo signaling, the core of which is a multiplecomponent kinase cascade that induces phosphorylation and suppression of downstream transcriptional activators, namely YAP and TAZ ${ }^{[5-7]}$. 
The deregulation of YAP and TAZ or other Hippo pathway components will cause diseases including various cancers ${ }^{[8,9]}$. p38 MAPK signaling is another highly conserved pathway that governs cell growth and death. This signaling negatively regulates the progression of cell cycle both in G1/M and G2/S transitions. Besides, it also mediates various stress-induced apoptosis ${ }^{[10,11]}$. Moreover, there are some reports suggesting that $\mathrm{p} 38$ can regulate the differentiation of various cells ${ }^{[12-14]}$. Despite such reports, the functions of both Hippo and p38 signaling in bladder cancer cells are still unclear.

In this study, we demonstrate how TAZ regulate the proliferation and apoptosis of bladder cancer cells. We found that the regulation of bladder cancer cells by Hippo pathway TAZ was mediated by p38 activity, which expanded our understanding of how signaling regulates cell proliferation and apoptosis.

\section{Methods}

\section{Antibody and Inhibitor}

Commercial TAZ antibodies were purchased from Abcam. p38, p-p38, caspase 3 antibodies and ccaspase 3 were purchased from Cell Signaling. Actin and Flag antibodies were obtained from Sigma. $\mathrm{PH}-$ 797804, a p38 inhibitor, was purchased from Selleck.

\section{Cell Culture}

All cell lines were cultured in an atmosphere of $5 \% \mathrm{CO}_{2}$ at $37^{\circ} \mathrm{C}$. T24 and 5637 cells were cultured in RPMI-1640 (Invitrogen) supplemented with 10\% FBS (Thermo Scientific), penicillin (100 U/ml) and streptomycin $(100 \mathrm{mg} / \mathrm{ml})$. 293T cells were cultured in DMEM (Invitrogen) supplemented with 10\% FBS (Thermo Scientific), penicillin $(100 \mathrm{U} / \mathrm{ml})$ and streptomycin $(100 \mathrm{mg} / \mathrm{ml})$.

\section{Plasmids and Lentiviruses Production}

The constructs for shCtrl and shTAZ expression in T24 and 5637 cells were prepared by cloning to negative control shRNA and TAZ shRNA sequences into a pLKO.1-puro lentiviral vector (Addgene \#8453) in line with the manufacturer's protocol. The sequence of the control shRNA was:

CCTATTTATGAGGCGACGGAA, and that of shTAZ was: AGAGGTACTTCCTCAATCA. For the stable overexpression of TAZ protein, TAZ cDNA was subcloned from a pEF-TAZ-N-Flag construct (Addgene \#19025) to pBABE-hygro (Addgene \#1765).

ShRNA-expressing lentiviruses were generated as follows. Briefy, for the construction of lentiviral shRNAexpressing vectors, 293T packaging cells were transfected by $6 \mu \mathrm{g}$ of each construct through lipofectamine reagents (Life Technologies, Grand Island, NY, USA). The lentivirus-containing supernatants were harvested $72 \mathrm{~h}$ after the transfection, and the lentiviruses with titers exceeding $1 \times 10^{7} \mathrm{CFU} / \mathrm{ml}$ were used for infection.

\section{Transfection}


On the day before the transduction, $3.5 \times 10^{5}$ cells were cultured in $500 \mu \mathrm{l}$ of cell growth medium, which was placed on 24-well plates. The cells should exhibit a 50-70\% confluence during transduction. The culture medium was replaced with a medium-containing polybrene and lentiviral particles, with the final concentration of polybrene reaching $8 \mu \mathrm{g} / \mathrm{ml}$. Then, the cells were incubated overnight before the lentivirus/polybrene mixture was replaced with fresh culture medium.

\section{RNA Extraction, Reverse Transcription (RT) and Quantitative Real-time PCR Analysis}

The cultures were harvested in Trizol (Invitrogen) for total RNA extraction, while the contaminated DNA was removed by DNase treatment. Total RNA was reversely transcribed into a complementary DNA through ReverTra Ace Qpcr RT Master Mix with gDNA removers (TOYOBO). The complementary DNA was then diluted and applied for quantification through real-time PCR, which was performed using SYBR Green Realtime PCR Master Mix (TOYOBO) and the Quantastudio3 Real-time PCR System (Applied Biosystems). The experiment was performed for at least twice, with duplicate biological replicates. PCR oligo sequences were purchased from Sangon Biotech: GAPDH (forward: AGCCACATCGCTCAGACAC; reverse: GCCCAATACGACCAAATCC), TAZ (forward: GGCTGGGAGATGACCTTCAC; reverse:

CTGAGTGGGGTGGTTCTGCT), CTGF (forward: CCAATGACAACGCCTCCTG; reverse:

TGGTGCAGCCAGAAAGCTC), CYR61 (forward: AGCCTCGCATCCTATACAACC; reverse:

TTCTTTCACAAGGCGGCACTC), ANKRD1 (forward: CACTTCTAGCCCACCCTGTGA; reverse:

CCACAGGTTCCGTAATGATTT), p38 (forward: CGTGTTGCAGATCCAGACCA; reverse: GCCAGAATGCAGCCTACAGA).

\section{Cell Proliferation and Viability Assay}

The T24 and 5637 cells infected with the virus carrying shCtrl or shTAZ vector were cultured in a 96 well plate $(2,000$ cells/well), whose number was measured on a daily basis by a Cell Counting Kit8 (CCK8) assay (Dojindo Molecular Technologies, Inc., Kumamoto, Japan) for 2 days. T24 and 5637 cells were cultured in 24-well plates. Cell growth was measured by means of an EdU incorporation assay in accordance with the product protocol. Briefly, T24 and 5637 cells were cultured in RPMI-1640 for 2 hours with EdU labeling. Then, the cells were fixed, permeated and stained by EdU solution. Cell nuclei were labeled by Hoechst 33342 (RiboBio, Guangzhou, China). Lastly, the treated cells were observed in a laser scanning microscope before being treated with $\mathrm{PH}-797840(30 \mu \mathrm{M})$ for 2 hours for subsequent cell proliferation and viability assay.

\section{Statistical Analysis}

Statistical analyses were performed using SPSS 21.0 (IBM SPSS, Armonk, NY, USA). P $<0.05$ was considered to indicate a statistical difference, and $\mathrm{P}<0.001$ was considered to indicate a statistically significant difference.

\section{Results}




\section{Knockdown of TAZ Expressions by shRNA Vectors}

TAZ is an important downstream effector of Hippo pathway. Up to now, however, it is still inconclusive whether the function of TAZ is related to bladder cancer cells. To study the function of TAZ in bladder cancer cells, the shRNA vector containing sequence-targeting TAZ coding region and the control shRNA were purchased. Firstly, RT-qPCR was performed to confirm the efficiency of TAZ shRNA. As shown in Fig. 1, the level of TAZ mRNA was significantly down-regulated when the cells were transfected with TAZ shRNA vectors (indicated as shTAZ). Since TAZ was a downstream factor of Hippo pathway, the knockdown of TAZ was supposed to affect the expression of target genes in the Hippo pathway. RT-qPCR was performed to examine several well-known Hippo target genes, including CTGF, CYR61 and ANKRD1. According to the result, the expression of all the three genes was down-regulated when TAZ expression was knocked down, thus further confirming the efficiency of TAZ shRNA.

\section{Knockdown of TAZ Inhibits Cell Proliferation}

Next, to establish whether TAZ could regulate the proliferation of bladder cancer cells, a cell-counting experiment was performed. Two bladder cancer cell lines, namely T24 cell and 5637 cell, were infected with virus-containing TAZ shRNA or negative control shRNA vectors. The total cell number was counted every 24 hours from Day 0 to Day 2. As shown in Fig. 2A-B, when TAZ expression was knocked down, T24 and 5637 cell lines containing shTAZ vectors grew at a slower pace than those containing shCtrl vectors, suggesting the significant reduction to TAZ-inhibited bladder cancer cells. The reduced cell growth might result from cell proliferation or apoptosis. To study how cell growth was inhibited by TAZ shRNA, cell proliferation was first analyzed after EdU staining. T24 and 5637 cells were infected with virus-containing control shRNA or TAZ shRNA (Fig. 2C-H). Compared to the control group, the cells expressing TAZ shRNA showed a decreased EdU positive ratio, which further supported that TAZ was necessary for the proliferation of bladder cancer cells.

\section{Knockdown of TAZ Induces Apoptosis}

To examine cell apoptosis, the expression of cleaved-caspase 3 was analyzed through western blot (Fig. 3A). The level of increased caspase3 protein was induced by the knockdown of TAZ. Additionally, the activated caspase3 (cleaved-caspase3) increased significantly when TAZ was knocked down, which clearly indicated that the loss of TAZ induced apoptosis.

\section{TAZ Regulates Stability of p38 Protein}

A further concern was raised about how TAZ regulated cell proliferation and apoptosis. To answer this question, the activity of several signaling pathways related to cell proliferation and apoptosis was tested, which led to the finding that the loss of TAZ contributed to an up-regulated level of p38 protein as well as phosphorylation, as shown in Fig. 3B. It is noteworty that the stability of p38 protein might be regulated by TAZ. To confirm this, Flag tagged TAZ in 293T cells was overexpressed to check the level of p38 protein. As shown in Fig. 3C, when TAZ was overexpressed, p38 protein was reduced clearly. Also, qPCR 
was performed to reveal that the over-expression of TAZ had no evident effect on the level of p38 mRNA (Fig. 3E-F). These results suggested that TAZ might regulate the stability of p38 protein.

\section{p38 Activity Mediates Regulation of Proliferation and Apoptosis through TAZ}

Though p38 signaling plays a vital role in cell proliferation and apoptosis, there is still no evidence suggesting that the function of TAZ is performed via p38 activity. To verify whether the effect of TAZ knockdown was produced through p38 signaling, PH-797804, a p38 inhibitor, was used to block p38 activity in the context of TAZ knockdown. The upregulation of cleaved-caspase 3 induced by TAZ knockdown disappeared when treated with $\mathrm{PH}-797804$ (Fig. 3D). This result suggested that p38 activity might mediate the apoptosis of TAZ-knockdown-induced cells.

A cell proliferation assay was further performed. In CCK assay, the treatment of $\mathrm{PH}-797804$ would not affect cell expressing control shRNA. However, for those expressing TAZ shRNA, cell proliferation ratio was increased by the treatment of PH-797804 compared to those of DMSO (Fig. 4A). In EdU staining assay, the reduction to EdU positive cells induced by TAZ knockdown was reversed by $\mathrm{PH}-797804$ treatment as well (Fig. 4B-F). Together, these results suggested that p38 activity mediated both cell proliferation and apoptosis regulated by TAZ knockdown.

\section{Discussion}

Both Hippo and p38 signaling played a crucial role in cell proliferation and apoptosis. However, whether they had a functional interaction in bladder cancer cells was not tested. In this report, it was found out that TAZ was required for the proliferation of bladder cancer cells and that the loss of TAZ significantly induced proliferation inhibition and increased apoptosis. It was also found out that TAZ might regulate the stability of p38 protein, and that TAZ knockdown induced proliferation inhibition and increased apoptosis via p38 activity. Interestingly, a report suggested that YAP, a TAZ homologue, could interact with p38 and suppress BTRC-induced p38 degradation, thus playing oncogenic roles in liver cancer cells

[15]. In this study, we have not identified the direct interaction between TAZ and p38. TAZ was a transcribed co-activator that regulated gene expression through the interaction with TEAD. Besides, it was reported that the use of bacterially-purified proteins revealed a direct interaction between p38 and TEAD in vitro ${ }^{[16]}$.

\section{Conclusions}

In this study, it was found out that TAZ could regulate the proliferation and apoptosis of bladder cancer cells. It was also discovered that TAZ might regulate the stability of p38 protein, the level of which was increased by the knockdown of TAZ while it was sharply reduced by the over-expressions of TAZ. Finally, it was found out that the regulation of bladder cancer cells by TAZ was mediated by p38 activity. The 
functional interaction between Hippo and p38 signaling was revealed in our work, which expanded our understanding of how signaling regulates cell proliferation and apoptosis.

\section{Abbreviations}

TAZ: WW domain containing transcription regulator 1

YAP1: Yes-associated protein 1

CTGF: Connective tissue growth factor

CYR61: Cysteine-rich angiogenic inducer 61

ANKRD1: Ankyrin repeat domain-containing protein 1

MAPK: Mitogen-activated protein kinase

DMSO: Dimethyl sulfoxide

FBS: Fetal bovine serum

RT-qPCR: Quantitative reverse transcription polymerase chain reaction

\section{Declarations}

Ethics approval and consent to participate

Not applicable.

\section{Consent for publication}

Not applicable.

\section{Availability of data and materials}

The datasets used and/or analysed during the current study are available from the corresponding author on reasonable request.

\section{Competing interests}

The authors declare that they have no competing interests

\section{Funding}

This work was supported by the National Key R\&D Program of China (2019YFA0906000), National Natural Science Foundation of China (81772737 and 81602223), Shenzhen Municipal Government of 
China (JCYJ20170413161749433) and the Sanming Project of Shenzhen Health and Family Planning Commission (SZSM201412018, SZSM201512037).

\section{Authors' contributions}

Conceptualization, Wei Chen and Weiren Huang; Data curation, Yangyang Sun and Lianxin Hu; Funding acquisition, Lianxin Hu and Weiren Huang; Methodology, Yangyang Sun and Lianxin Hu; Validation, Yangyang Sun, Lianxin Hu, Chengxi Liu, Ganglin Su and Lina Yang; Writing - original draft, Yangyang Sun, Lianxin Hu, Chengxi Liu, Ganglin Su and Lina Yang; Writing - review \& editing, Wei Chen and Weiren Huang. All authors have read and agreed to the published version of the manuscript.

\section{Acknowledgements}

Not applicable.

\section{References}

1. Berdik C. Unlocking bladder cancer. Nature. 2017; 551(7679): S34-S35.

2. Rebecca LS, Kimberly DM and Ahmedin J. Cancer statistics, 2020. CA: A Cancer Journal for Clinicians. 2020; 70(1): 7-30.

3. Racioppi M, D'Agostino D, Totaro A, et al. Value of current chemotherapy and surgery in advanced and metastatic bladder cancer. Urol Int. 2012; 88(3): 249-258.

4. Soloway MS. Bladder cancer: Lack of progress in bladder cancer-what are the obstacles? Nat Rev Urol. 2013; 10(1): 5-6.

5. Yu FX, Zhao B and Guan KL. Hippo Pathway in Organ Size Control, Tissue Homeostasis, and Cancer. Cell. 2015; 163(4): 811-828.

6. Plouffe SW, Hong AW and Guan KL. Disease implications of the Hippo/YAP pathway. Trends Mol Med. 2015; 21(4): 212-222.

7. Tang Y, Fang G, Guo F, et al. Selective Inhibition of STRN3-Containing PP2A Phosphatase Restores Hippo Tumor-Suppressor Activity in Gastric Cancer. Cancer Cell. 2020; 38(1): 115-128.

8. Barron DA and Kagey JD. The role of the Hippo pathway in human disease and tumorigenesis. Clin Transl Med. 2014; 3: 1-25.

9. Harvey KF, Zhang X and Thomas DM. The Hippo pathway and human cancer. Nat Rev Cancer. 2013; 13(4): 246-257.

10. Wagner EF and Nebreda AR. Signal integration by JNK and p38 MAPK pathways in cancer development. Nat Rev Cancer. 2009; 9(8): 537-549.

11. Bradham C and McClay DR. p38 MAPK in development and cancer. Cell Cycle. 2006; 5(8): 824-828.

12. Ventura JJ, Tenbaum S, Perdiguero E, et al. p38a MAP kinase is essential in lung stem and progenitor cell proliferation and differentiation. Nature Genetics. 2007; 39(6): 750-758. 
13. Aouadi M, Bost F, Caron L, et al. p38 Mitogen-Activated Protein Kinase Activity Commits Embryonic Stem Cells to Either Neurogenesis or Cardiomyogenesis. Stem Cells. 2006; 24(5): 1399-1406.

14. Schmelter M, Ateghang B, Helmig S, et al. Embryonic stem cells utilize reactive oxygen species as transducers of mechanical strain-induced cardiovascular differentiation. Faseb Journal Official Publication of the Federation of American Societies for Experimental Biology. 2006; 20(8): 11821184.

15. Wang JY, Ma LF, Weng WH, et al. Mutual interaction between YAP and CREB promotes tumorigenesis in liver cancer. Hepatology. 2013; 58: 1011-1020.

16. Lin KC, Moroishi T, Meng Z, et al. Regulation of Hippo pathway transcription factor TEAD by p38 MAPK-induced cytoplasmic translocation. Nat Cell Biol. 2017; 19(8): 996-1002.

\section{Figures}
A
B

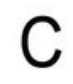
C

TAZ

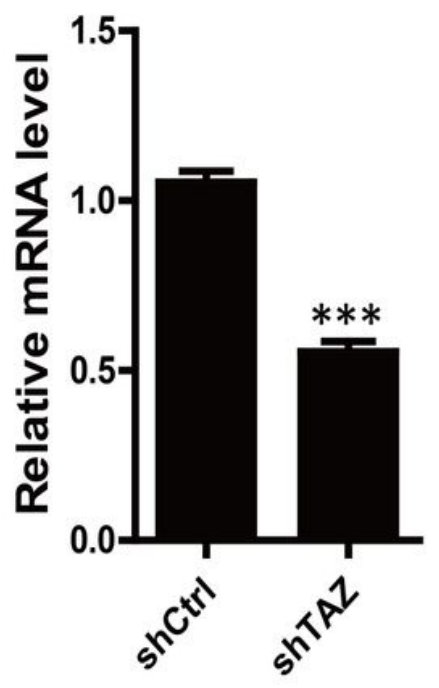

CTGF

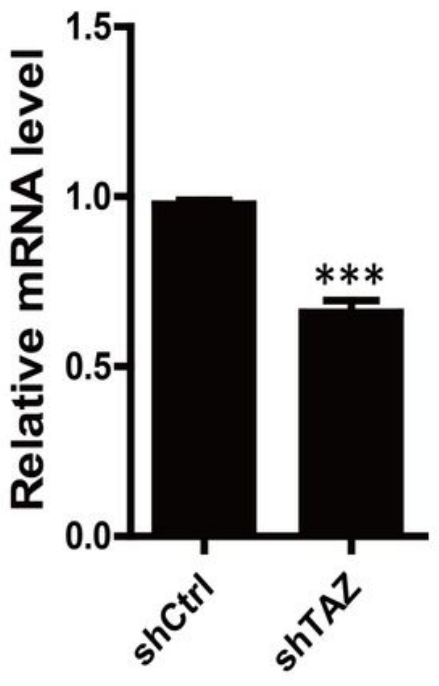

D

ANKRD1
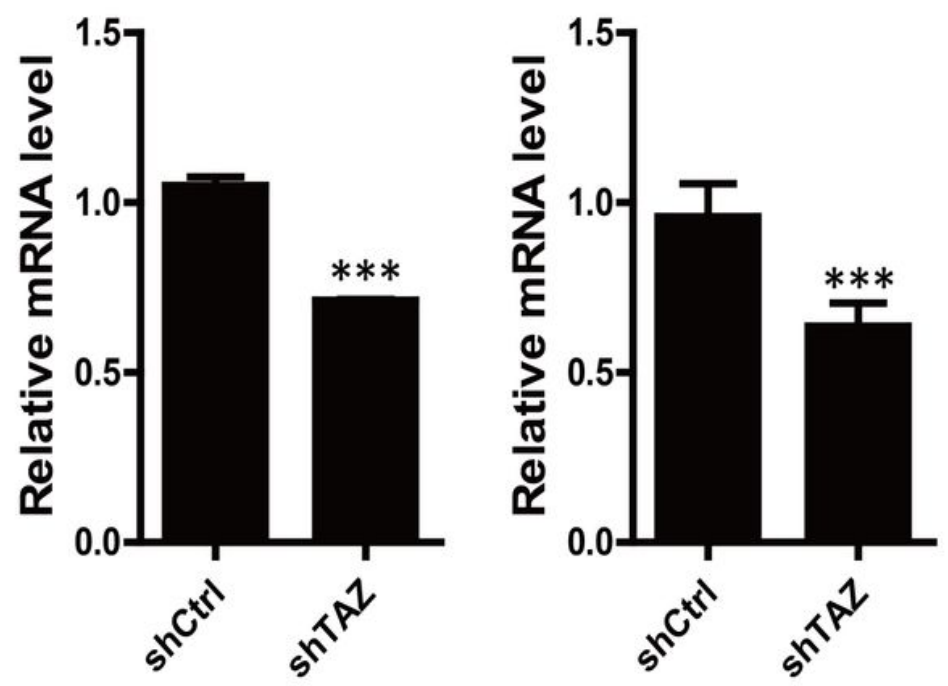

Figure 1

Confirm efficiency of TAZ knockdown. (A-D) T24 cells were transfected with control or TAZ shRNA vectors, and RT-qPCR was performed to analyze mRNA level of indicated genes. The results were shown as mean $\pm S D, N=3$. *** $P<0.001$. 

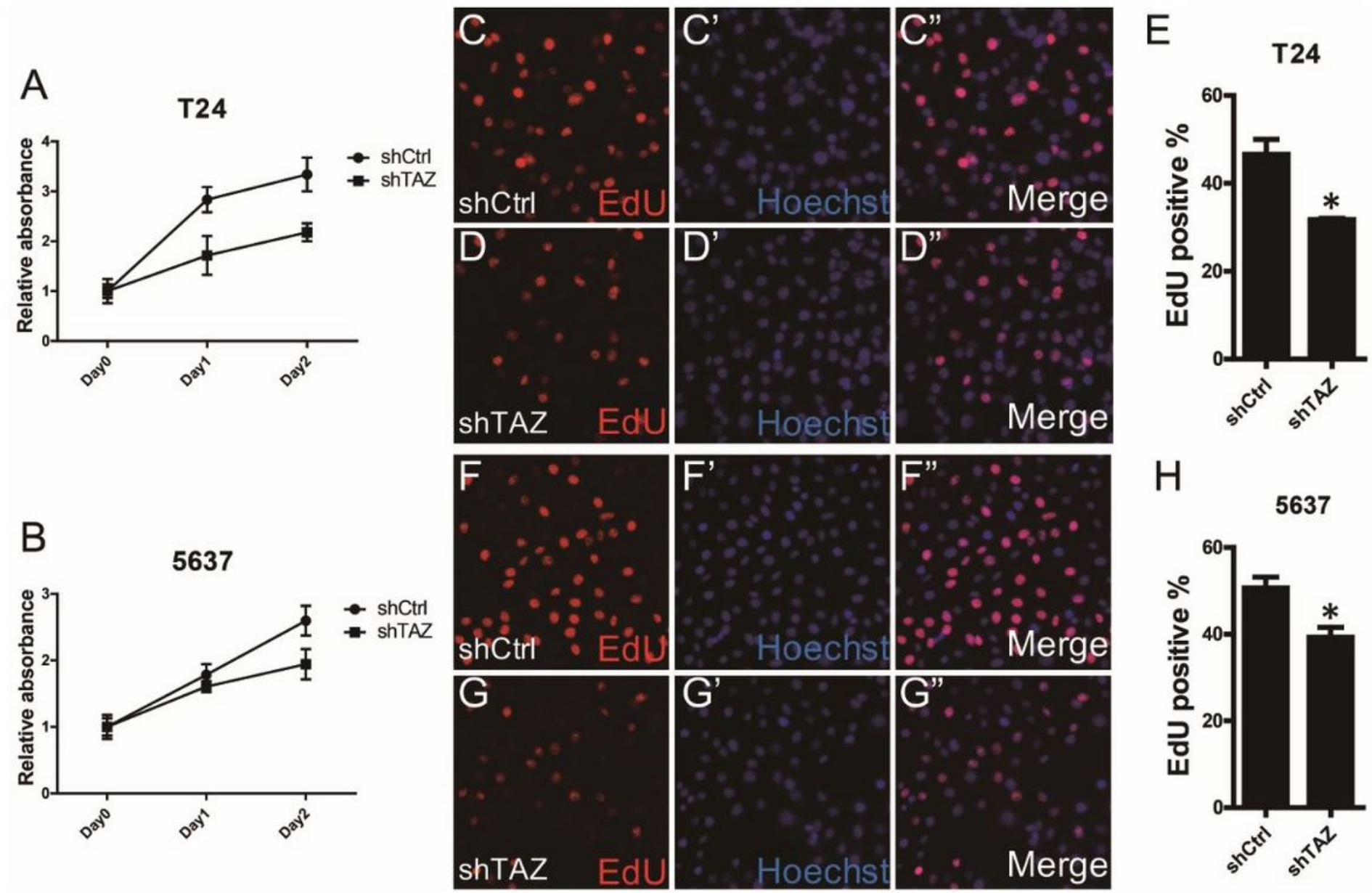

$\mathrm{H}$

5637

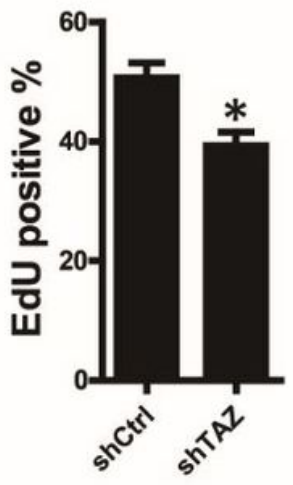

Figure 2

Knockdown of TAZ inhibits proliferation of bladder cancer cells. (A-B) Bladder cancer cell lines T24 (A) and 5637 (B) were infected with virus-carrying shCtrl or shTAZ vectors. CCK experiments were performed at indicated time points to analyze cell proliferation. (C-E) T24 cells were infected with virus-carrying shCtrl or shTAZ vectors, and then subjected for EdU assay. Representative pictures of EdU assay were shown (C-D"). Hoechst was used to show cell nuclei. The ratio of EdU positive cells was calculated (E). The result was shown as mea $n \pm S D, N=3$. * $P<0.05$. $(F-H) 5637$ cells were infected with virus-carrying shCtrl or shTAZ vectors, and then subjected for EdU assay. Representative pictures of EdU assay were shown (F-G"). Hoechst was used to show cell nuclei. The ratio of EdU positive cells was calculated $(\mathrm{H})$. The result was shown as mean $\pm S D, N=3$. * $P<0.05$. 
A

B

严

c-caspase 3

p-p38

caspase3

TAZ

Actin

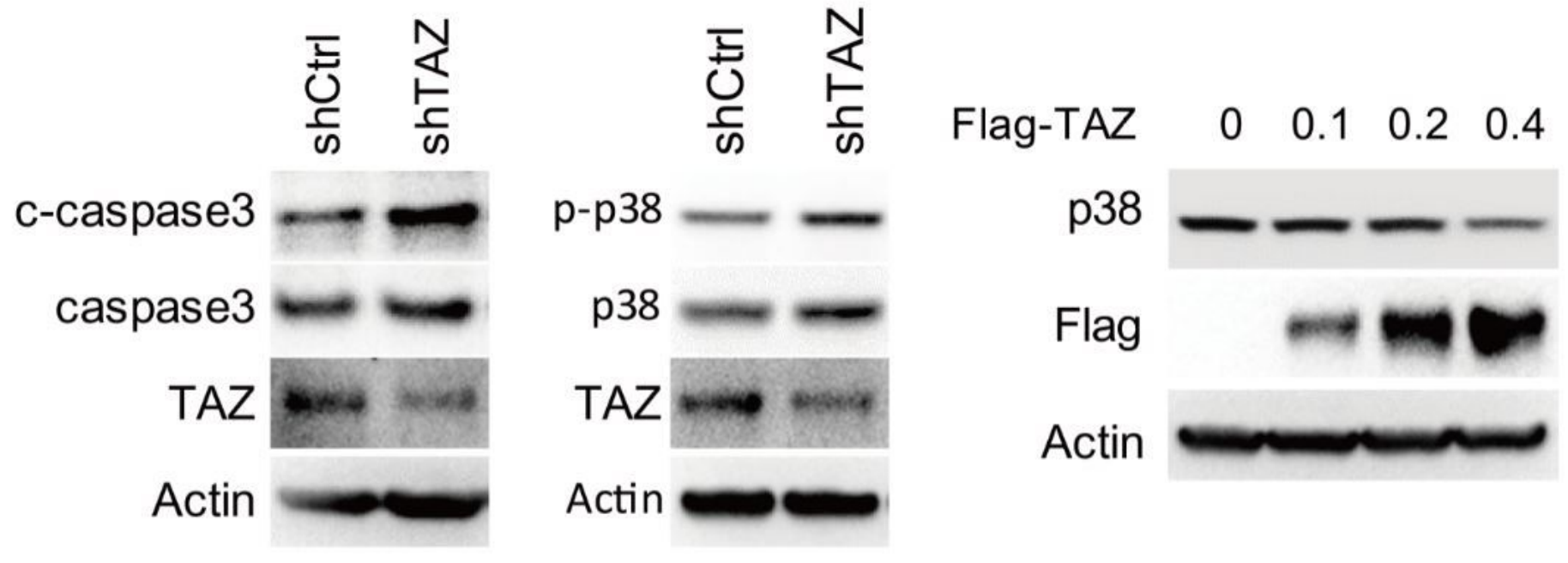

Flag-TAZ

C
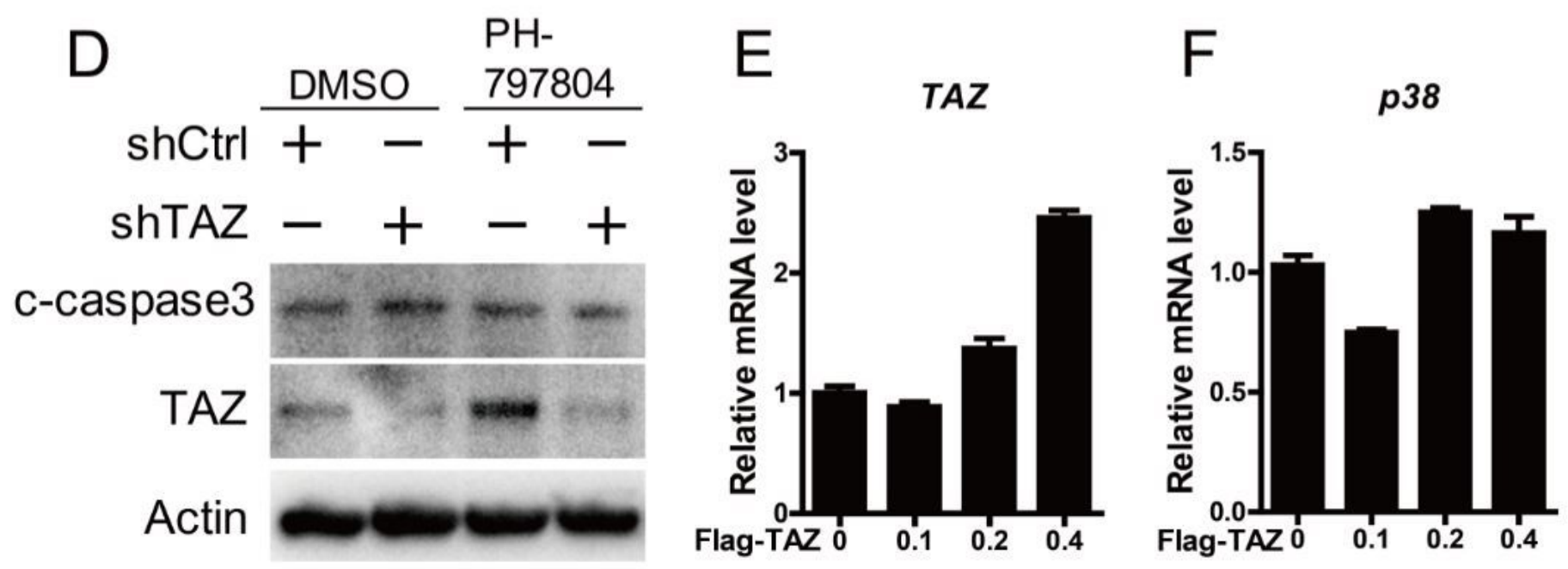

Figure 3

TAZ may regulate p38 stability and p38-dependent cell apoptosis. (A) Cell apoptosis was increased by knockdown of TAZ. T24 cells were infected with virus-carrying shCtrl or shTAZ vectors and then subjected for western blot analysis with indicated antibodies. (B) Levels of p38 protein and phosphorylation were increased by knockdown of TAZ. T24 cells were infected with virus-carrying shCtrl or shTAZ vectors and then subjected for western blot analysis with indicated antibodies. (C) Level of p38 protein was downregulated by overexpression of TAZ. T24 cells were transfected with different amounts of Flag-TAZ plasmids and then subjected for western blot analysis with indicated antibodies. (D) p38-dependent apoptosis was induced by knockdown of TAZ. T24 cells were infected with virus-carrying shCtrl or shTAZ vectors and treated with DMSO or PH-797804, and then subjected for western blot analysis with indicated antibodies. (E-F) Overexpression of TAZ did not affect level of p38 mRNA. T24 cells were transfected with different amounts of Flag-TAZ plasmids, and then RT-qPCR was performed to analyze levels of TAZ (E) and p38 (F) mRNA. 


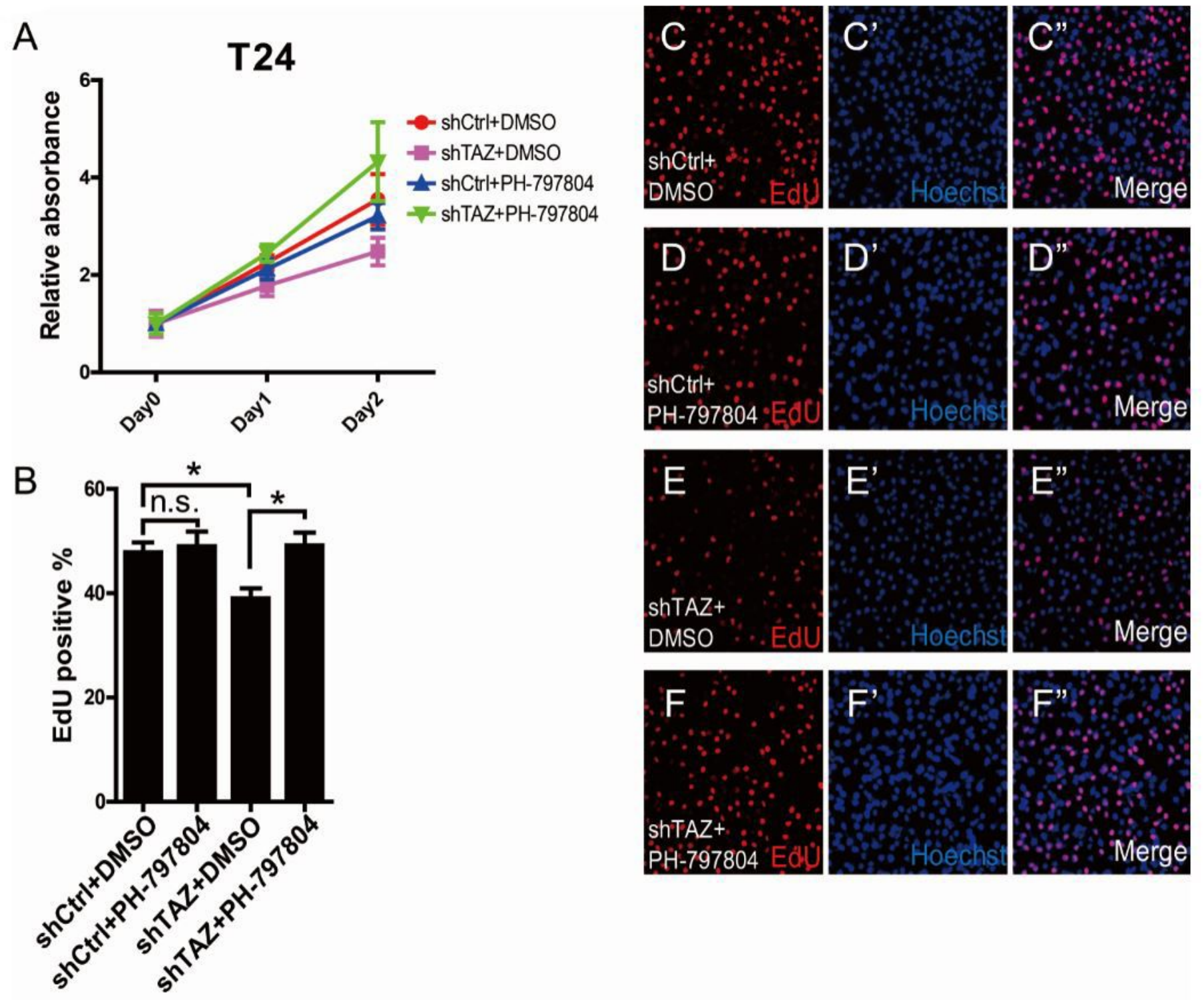

Figure 4

TAZ regulated proliferation of bladder cancer cells via p38 activities. (A) p38 activities mediated TAZknockdown-induced cell proliferation. T24 cells were infected with virus-carrying shCtrl or shTAZ vectors and treated with DMSO or $\mathrm{PH}-797804$. CCK experiments were performed at indicated time points to analyze cell proliferation. (B-F") T24 cells were infected with virus-carrying shCtrl or shTAZ vectors and treated with DMSO or $\mathrm{PH}-797804$, and then subjected for EdU assay. Representative pictures of EdU assay were shown (C-F"). Hoechst was used to show cell nuclei. The ratio of EdU positive cells was calculated $(B)$. The result was shown as mean $\pm S D, N=3 .{ }^{*} P<0.05$. n.s. no significant difference.

\section{Supplementary Files}

This is a list of supplementary files associated with this preprint. Click to download.

- rawdata.pdf 